\title{
Long non-coding RNA MEG3 induces cell apoptosis in esophageal cancer through endoplasmic reticulum stress
}

\author{
ZHEN-LUN HUANG ${ }^{1}$, RUI-PEI CHEN ${ }^{1}$, XIAO-TAO ZHOU ${ }^{1}$, HAO-LIAN ZHAN ${ }^{1}$, \\ MIN-MIN HU ${ }^{1}$, BIN LIU ${ }^{2}$, GUAN-DI WU ${ }^{3}$ and LING-FEI WU ${ }^{1}$ \\ Departments of ${ }^{1}$ Gastroenterology, and ${ }^{2}$ Neurosurgery, Second Affiliated Hospital, \\ Shantou University Medical College, Shantou, Guangdong 515041; ${ }^{3}$ First Clinical Medical College, \\ Southern Medical University, Guangzhou, Guangdong 510515, P.R. China
}

Received November 1, 2016; Accepted March 27, 2017

DOI: $10.3892 /$ or.2017.5568

\begin{abstract}
Long non-coding RNAs (lncRNAs) play important roles in diverse biological processes, such as cell growth, apoptosis and migration. Although downregulation of lncRNA MEG3 has been identified in several cancers, little is known about its role in esophageal squamous cell carcinoma (ESCC). The aim of the present study was to detect MEG3 expression in clinical ESCC tissues, investigate its biological functions and the endoplasmic reticulum (ER) stress-relative mechanism. MEG3 expression levels were detected by qRT-PCR in both tumor tissues and adjacent non-tumor tissues from 28 ESCC patients. pcDNA3.1-MEG3 recombinant plasmids were constructed and transfected to EC109 cells. Cell growth was analyzed by CCK-8 assay. Cell apoptosis was analyzed by fluorescence microscope and Annexin V/PI assay. The protein expression was determined by western blot analysis. The results showed that MEG3 decreased significantly in ESCC tissues relative to adjacent normal tissues. pcDNA3.1-MEG3 plasmids were successfully constructed and the expression level of MEG3 significantly increased after MEG3 transfection to EC109 cells. Ectopic expression of MEG3 inhibited EC109 cell proliferation and induced apoptosis in vitro. MEG3 overexpression increased the expression of ER stress-related proteins (GRP78, IRE1, PERK, ATF6, CHOP and cleaved-caspase-3). Our results first demonstrate that MEG3 is downregulated in ESCC tissues. MEG3 was able to inhibit cell growth and induced apoptosis in EC109 cells, most probably via activation of the ER stress pathway.
\end{abstract}

Correspondence to: Professor Ling-Fei Wu, Department of Gastroenterology, Second Affiliated Hospital, Shantou University Medical College, Shantou, Guangdong 515041, P.R. China E-mail: 1808435253@qq.com

Key words: lncRNA MEG3, esophageal squamous cell carcinoma, EC109 cell, endoplasmic reticulum stress, apoptosis

\section{Introduction}

Esophageal cancer is a main cancer in China and the sixth leading cause of cancer-related death worldwide, with considerable geographical variations in incidence (1). Shantou is a high-risk area of esophageal squamous cell carcinoma. The clinicopathological features of ESCC are different from the esophageal adenocarcinoma in Western countries (2). The molecular mechanisms of esophageal cancer remain unclear. According to encode project, $\sim 80 \%$ of the human genome is transcribed to RNA, of which $\sim 90 \%$ genome transcribes into non-coding RNA. These non-coding RNAs are increasingly recognized and defined as endogenous regulatory RNA involved in maintenance of cellular homeostasis during growth, development and pathogenesis disease $(3,4)$. Maternally expressed gene 3 (MEG3), which is an imprinted gene, located on chromosome 14q32. MEG3 was first identified as the ortholog of gene trap locus 2 (Gtl2) in mice (5). It is an imprinted gene expressed from the maternal allele with a length of $\sim 1.6-\mathrm{kb}$ nucleotides. Although MEG3 is expressed in many human normal tissues, the loss of MEG3 expression has been found in various types of human tumors, including non-small cell lung cancer, hepatoma, gastric, prostate and other cancers (6-9). Furthermore, MEG3 can inhibit cancer cell proliferation or induce apoptosis in vitro and in vivo by stimulating p53-dependent transcription (10-12).

Endoplasmic reticulum (ER) is the site of protein synthesis, protein folding, maintainance of calcium homeostasis, synthesis of lipids and sterols (13). Various stimuli can disturb ER homeostasis and result in the accumulation of unfolded or misfolded proteins leading to pathological consequences, generally known as 'ER stress'. Homeostatic regulation in the ER is under the control of three evolutionary conserved pathways: IRE1a-XBP-1, PERK-eIF2a and ATF6. These pathways, also referred to as the unfolded protein response (UPR), are activated in response to ER stress (14). UPR promotes cell adapt stress. However, when the response is not sufficient and ER stress persists, UPR changes its function from acting to promote cellular survival to commit the cell to apoptosis through upregulation of pro-apoptotic factors, such as CHOP and caspase-4/12 (15). 
Although the expression of MEG3 has been shown to be downregulated in some tumor tissues, the biological role of MEG3 and its mechanism in ESCC remain largely unknown. Especially, very little is known about the regulation relationship between MEG3 and ER stress in ESCC. In the present study, we characterized the expression of MEG3 in ESCC tissues and adjacent normal esophageal tissues and explored its effects on EC109 cells.

\section{Materials and methods}

Clinical samples and tissue processing. Twenty-eight paired esophageal cancer tissues and adjacent normal tissues were obtained from patients who underwent surgery at the Second Affiliated Hospital of Shantou University Medical College between 2011 and 2015. Among them, 20 males and 8 females, aged from 42 to 75 years. No local or systemic treatment was conducted in these patients before surgery. All of the specimens were collected within 30 min after tumor resection and stored in liquid nitrogen immediately until RNA extraction. Written consents were obtained from all patients prior to surgery and the study protocol was approved by the Ethics Committee of the Second Affiliated Hospital of Shantou Medical College.

Cell culture and experimental groups. EC109 cells were purchased from the Shanghai Institute of Biochemistry and Cell Biology (Shanghai, China). Cells were cultured in Dulbecco's modified Eagle's medium (DMEM) containing 10\% (v/v) fetal bovine serum (FBS; Invitrogen Life Technologies, Carlsbad, CA, USA), penicillin (final concentration, $100 \mathrm{U} / \mathrm{ml}$ ), and streptomycin (final concentration, $100 \mathrm{mg} / \mathrm{ml}$ ), under a humidified atmosphere with $5 \% \mathrm{CO}_{2}$ at $37^{\circ} \mathrm{C}$. The cell culture medium was changed every one or two days, and cells were passaged at $80-90 \%$ confluence. EC109 cells were transfected with pcDNA3.1 empty plasmids or pcDNA3.1-MEG3 plasmids and divided into two groups: pcDNA3.1 (control group, Ctrl) and pcDNA3.1-MEG3 (overexpressing MEG3 group, MEG3).

Plasmid identification and cell transfection and bacterial transformation. The MEG3 gene was designed and synthesized by means of PCR according to the GenBank of Human MEG3 (NR_002766) gene sequence as previously described (16). The MEG3 gene fragment was identified by restriction endonuclease analysis and inserted into a plasmid (pcDNA3.1). Plasmids containing MEG3 sequence were then transfected into competent cell DH5 $\alpha$. To obtain plasmids stably expressing MEG3, pcDNA3.1-MEG3 plasmids were extracted from E. coli (DH5 $\alpha)$.

For ectopic MEG3 expression, pcDNA3.1-MEG3 plasmids were transiently transfected into EC109 cells using Lipofectamine 2000 transfection reagent (Invitrogen Life Technologies, Shanghai, China) according to the manufacturer's instructions. pcDNA3.1 empty plasmids were transfected to cells in parallel as a control. RT-PCR was performed to examine mRNA expression level of MEG3.

RNA extraction and $q R T-P C R$ assay. Total RNA was extracted from tissues or cultured cells with TRIZol reagent (Invitrogen Life Technologies) according to the manufacturer's protocol.
The isolated RNA was reverse transcribed into cDNA using a reverse transcription kit (Takara Biotechnology, Dalian, China). The expression was quantified by qRT-PCR using a standard protocol from the SYBR-Green PCR kit (Toyobo, Co., Ltd., Osaka, Japan) on the StepOnePlus qRT-PCR System (Applied Biosystems, Foster City, CA, USA). The following gene specific primers: forward, 5'-CTGCCCATC TACACCTCACG-3' and reverse, 5'-CTCTCCGCCGTCTGC GCTAGGGGCT-3 for MEG3; forward, 5' GTCAACGGATT TGGTCTGTATT3' and reverse, 5'-AGTCTTCTGGGTGG CAGTGAT-3' for GAPDH. Forward, 5'-AAACGGCTACCA CATCCAAG-3' and reverse 5'-CAATTACAGGGCCTCGA AAG-3' for $18 \mathrm{~S}$. The PCR reaction was conducted at $95^{\circ} \mathrm{C}$ for $30 \mathrm{sec}$ followed by 40 cycles of $95^{\circ} \mathrm{C}$ for $5 \mathrm{sec}$ and $60^{\circ} \mathrm{C}$ for $30 \mathrm{sec}$. Each sample was analyzed in triplicate and the relative expression was calculated using the $2^{-\Delta \Delta \mathrm{Ct}}$ method relative to GAPDH or $18 \mathrm{~S}$.

Cell viability assay by $C C K-8$. The viability of cells transfected with pcDNA3.1-MEG3 or pcDNA3.1 was determined by using Cell Counting kit-8 (CCK-8). EC109 cells were inoculated into 96 -well plates at $5 \times 10^{3}$ cells/well for $24 \mathrm{~h}$. Cells were transfected with pcDNA3.1-MEG3 or pcDNA3.1 for $48 \mathrm{~h}$. The culture medium was then discarded and the cells were washed with phosphate-buffered saline (PBS), and then bred with $0.5 \mathrm{mg} /$ $\mathrm{ml}$ of 2-(2-methoxy-4-nitrophenyl)-3-(4-nitrophenyl)-5-(2,4disulfoanilino)-2H-tetrazolium monosodium salt (WST-8) at $37^{\circ} \mathrm{C}$ for $4 \mathrm{~h}$. After incubation, the concentration of product was determined by measuring the absorbance at $450 \mathrm{~nm}$ using an enzyme-linked immunosorbent assay reader (FilterMax F5; Molecular Devices, Sunnyvale, CA, USA).

Fluorescence microscope and flow cytometric analysis of apoptosis. Apoptotic nuclei staining. DAPI is a fluorescent dye which specifically conjugates to ds-DNA and is thus used to visualize nuclear morphological features; the nuclei of apoptotic cells demonstrate condensation and fragmentation. EC109 cells transfected with pcDNA3.1-MEG3 or pcDNA3.1 were harvested at $48 \mathrm{~h}$. Cells were fixed in $4 \%$ paraformaldehyde for $30 \mathrm{~min}$ at room temperature and then permeabilized with $0.2 \%$ Triton X-100 in PBS for $5 \mathrm{~min}$ at room temperature. DAPI $(500 \mathrm{ng} / \mathrm{ml})$ staining was performed at room temperature for $10 \mathrm{~min}$. The morphology of the nuclei was viewed and captured with a fluorescence microscope (Olympus BX51; Olympus, Tokyo, Japan).

EC109 cells transfected with pcDNA3.1-MEG3 or pcDNA3.1 were harvested at $48 \mathrm{~h}$. Cells were washed twice with ice-cold PBS and resuspended in $1 \mathrm{X}$ binding buffer to a concentration of $1 \times 10^{6} / \mathrm{ml}$. The cells were then bled with $5 \mu 1$ Annexin V/FITC and $10 \mu \mathrm{l}$ propidium iodide (PI) $(20 \mu \mathrm{g} / \mathrm{ml})$ for $15 \mathrm{~min}$ at room temperature keeping out of light. Analyses were performed with a BD Accuri ${ }^{\mathrm{TM}}$ C6 flow cytometer (BD Biosciences, San Diego, CA, USA) with the FL1 and FL3 detector.

Western blot analysis. EC109 cells transfected with pcDNA3.1-MEG3 or pcDNA3.1 were harvested at $48 \mathrm{~h}$ and washed with ice-cold PBS three times. The cellular protein lysates were prepared with RIPA buffer containing PMSF. Protein fractions were prepared using a nuclear and 


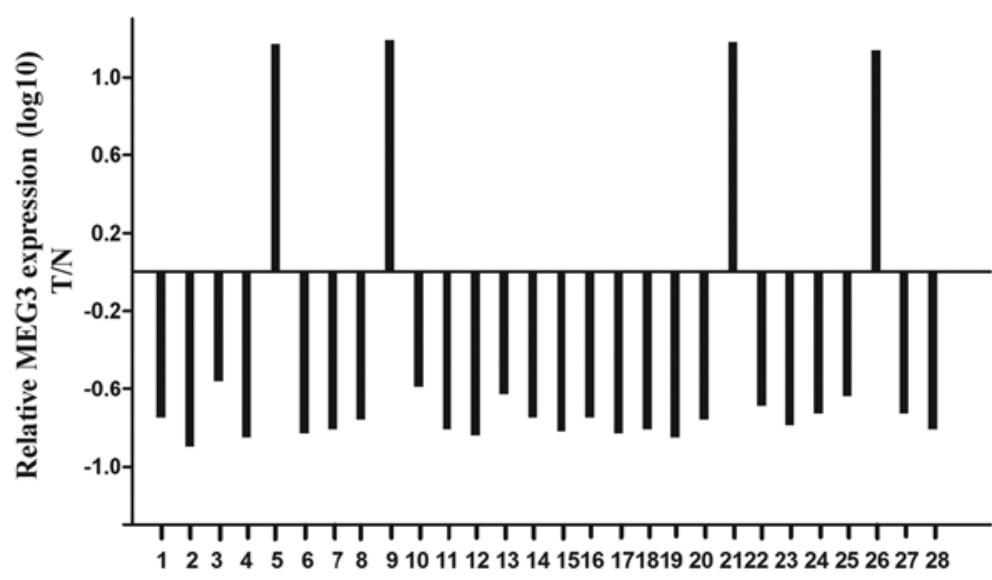

Sample number of ESCC

Figure 1. Relative MEG3 expression levels in esophageal cancer tissues. Analysis of the MEG3 expression level was carried out in esophageal cancer tissues and in paired adjacent normal tissues $(n=28)$. The relative fold-change was calculated by $2^{-\Delta \Delta C t}$ and the results were expressed as $\log 10$. GAPDH was used as the internal control. N, tumor tissue group, and T, non-tumor tissue group. In 28 patients, N/T ratio was negative in 24 cases.

cytoplasmic protein extraction kit (Beyotime Institute of Biotechnology, Haimen, China). Protein concentration was measured by using a BCA protein assay kit (Thermo Fisher Scientific, Waltham, MA, USA) according to the manufacturer's instructions. For western blot assay, protein $(50 \mu \mathrm{g})$ was subjected to electrophoresis on SDS-PAGE and then transfered onto nitrocellulose membranes. The membranes were blocked with $5 \%$ non-fat dry milk in Tris-buffered saline ( $\mathrm{pH}$ 7.6, TBS) containing $0.5 \%$ Tween-20 at room temperature for $60 \mathrm{~min}$. Then membranes were incubated with primary antibodies against: $\beta$-actin $(1: 5,000)$, GRP78 (1:500), ATF6 (1:500), IRE1 (1:500), PERK (1:500), CHOP $(1: 1,000)$, caspase-9 $(1: 1,000)$ and cleaved caspase-3 $(1: 1,000)$ in TBST at $4^{\circ} \mathrm{C}$ overnight. The next day, membranes then were washed with TBST and incubated with fluorescent secondary antibodies (LI-COR Biosciences, Lincoln, NE, USA) coupled to the first antibody at room temperature out of light for $1 \mathrm{~h}$, followed by TBST washing three times. Drying with neutral absorbent paper and scanned by Odyssey Detection System (LI-COR Biosciences). Expression of proteins was analyzed using the Quantity One software (Bio-Rad Laboratories, Hercules, CA, USA) and was normalized to that of $\beta$-actin (for total cell fraction).

Statistical analysis. All experiments were independently performed at least three times. The values are expressed as mean $\pm \mathrm{SD}$. The statistical significance of the data was calculated using Student's t-test, Chi-square test or one-way analysis of variance (ANOVA) with a post-hoc test of multiple comparisons. A $\mathrm{P}<0.05$ was considered statistically significantly.

\section{Results}

MEG3 expression is downregulated in human esophageal cancer tissues. To find whether lncRNA MEG3 was differentially expressed in the ESCC tissues, a total of 28 paired esophageal cancer tissues and adjacent normal tissues were analyzed for MEG3 expression using qRT-PCR and normalized to GAPDH. Our results showed that the expression level of MEG3 was significantly lower in ESCC tissues compared with the normal tissues (Fig. 1; $\mathrm{P}<0.05$ ), and the expression level of MEG3 was negatively correlated with prognosis (data not shown).

pcDNA3.1-MEG3 plasmid construction and identification. The MEG3 sequence was synthesized and subcloned into pcDNA3.1 (Invitrogen Life Technologies) to generate pcDNA3.1-MEG3. LncRNA MEG3 gene was confirmed by $X h o \mathrm{I}$ and $\mathrm{BamHI}$ restriction enzyme digestion, then inserted into the pcDNA3.1 vector. The particular sequence of MEG3 inserted into vectors was the same as designed. The fragment did not appear in the control vector, showing that pcDNA3.1-MEG3 plasmid was successfully constructed (Fig. 2).

MEG3 expression increases after pcDNA3.1-MEG3 transfection in EC109 cells. To explore the effect of MEG3 in EC in vitro, we first measured its expression in EC109 cell lines. EC109 cells were transiently transfected with either pcDNA3.1 empty plasmids (control) or pcDNA3.1-MEG3 (overexpressing MEG3). The expression levels of MEG3 mRNA were measured by RT-PCR. As shown in Fig. 3A, the expression level of MEG3 is low in normal EC109 cells. Compared to the blank group (cells were not treated by any plasmids) or control group (pcDNA3.1), the mRNA expression level of MEG3 was significantly increased after transfection with pcDNA3.1-MEG3. It demonstrated that pcDNA3.1-MEG3 was successfully transfected and high level mRNA expression of MEG3 was obtained in EC109 cells (Fig. 3).

Ectopic expression of MEG3 inhibits cell proliferation. Then, we attempted to determine whether MEG3 affects EC109 cell proliferation in vitro. Cell proliferation was measured by the CCK-8 assay. Ectopic expression of MEG3 significantly decreased cell vitality rate by $16.9 \%$ and $22.6 \%$ after 48 and $72 \mathrm{~h}$, respectively, compared with the control group (Fig. 4; both $\mathrm{P}<0.05)$. These results suggest that overexpressing MEG3 can inhibit EC109 cell growth. 
A

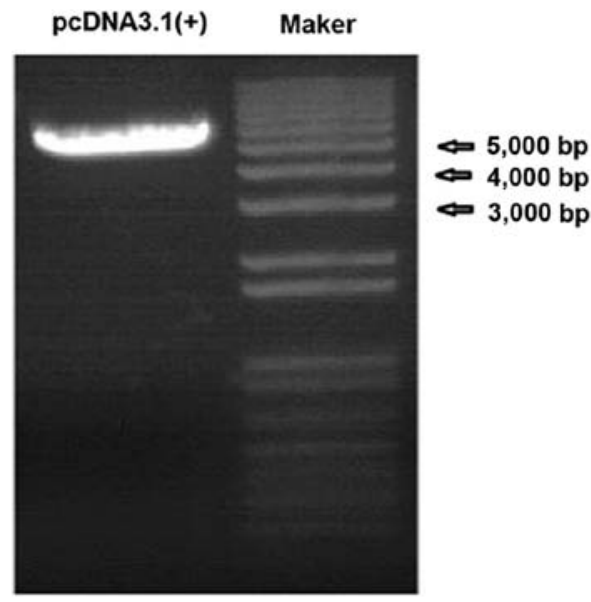

B pcDNA3.1-MEG3 Maker

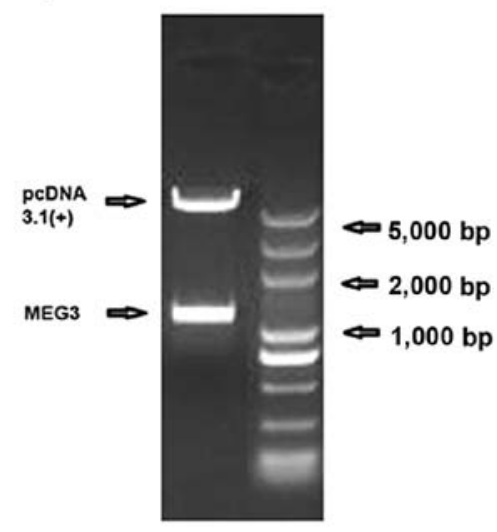

C

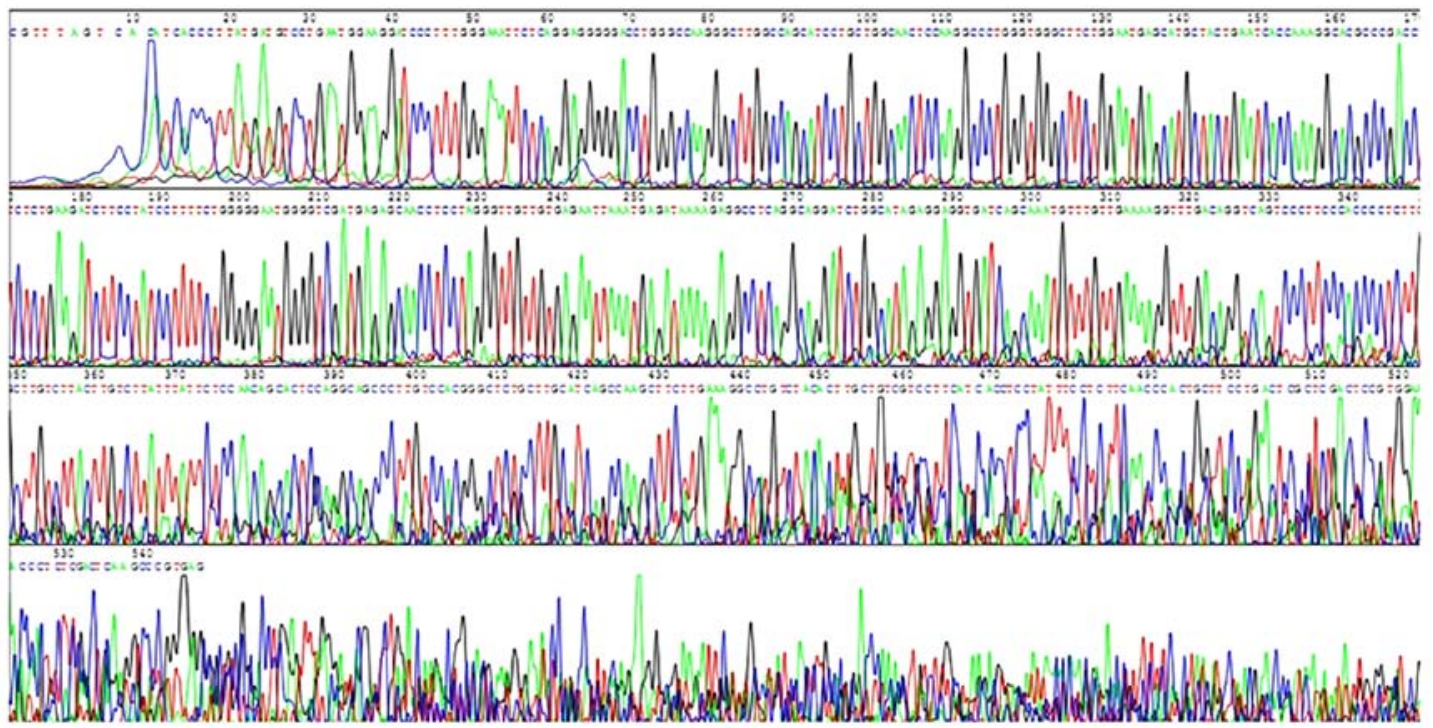

Figure 2. pcDNA3.1-MEG3 plasmid was successfully constructed. (A) Agarose gel electrophoresis of eukaryotic expression vector pcDNA 3.1(+). The molecular weight of pcDNA 3.1(+) is 5428 bp. (B) Agarose gel electrophoresis of fragment of MEG3 gene. The molecular weight of lncRNA MEG3 is 1595 bp. (C) Sequence diagram of inserted gene fragment. Sequence analysis confirmed that the sequence of MEG3 gene was successfully inserted into the constructed recombinant plasmid.

A

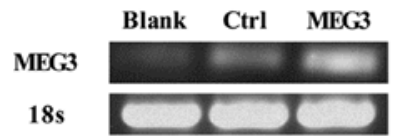

B

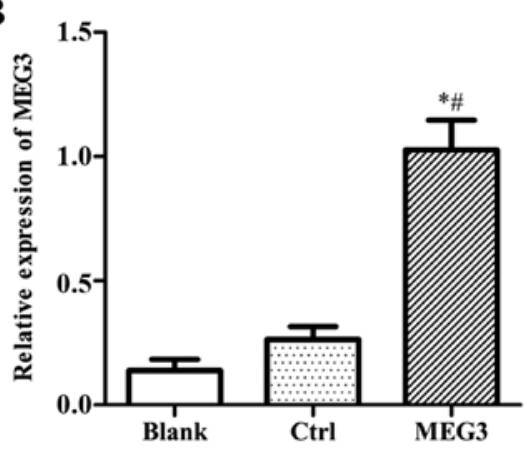

Figure 3. pcDNA3.1-MEG3 was successfully transfected and high level mRNA expression of MEG3 was obtained in EC109 cells. (A) The mRNA expression levels of MEG3 in blank (no plasmids), control (pcDNA3.1) and pcDNA3.1-MEG3 transfection group. (B) Quantitative analysis of the band intensity shown in (A). The bars represent mean \pm SD of at least three independent experiments. ${ }^{*} \mathrm{P}<0.05$ vs. blank group; ${ }^{\text {}} \mathrm{P}<0.05$ vs.control group.
Ectopic expression of MEG3 induces cell apoptosis. In order to clarify whether the inhibition of cell proliferation is related to cell death or apoptosis, DAPI staining and the flow cytometric (Annexin V/PI) assay were performed. The morphologic hallmarks of apoptosis include chromatin margination, nuclear condensation and fragmentation. As shown in Fig. 5A-MEG3, in overexpressing MEG3 cells, some cell nuclei became condensed and shrunken and typical apoptotic bodies appeared. However, normal cell nuclei were uniform and without condensation or fragmentation in the control group (Fig. 5A-Ctrl). Flow cytometric assay further confirmed the DAPI staining results. The apoptosis rate in MEG3 overexpression group is significantly higher than that of the control (Fig. 5B, 9.56 \pm 2.6 vs. $1.41 \pm 0.37 \%$; $\mathrm{P}<0.05$ ), showing that ectopic expression of MEG3 induces cell apoptosis.

Ectopic expression of MEG3 activates the ER stress pathway. To explore the mechanism by which MEG3 induced growth arrest and apoptosis, western blot assay was carried out to 


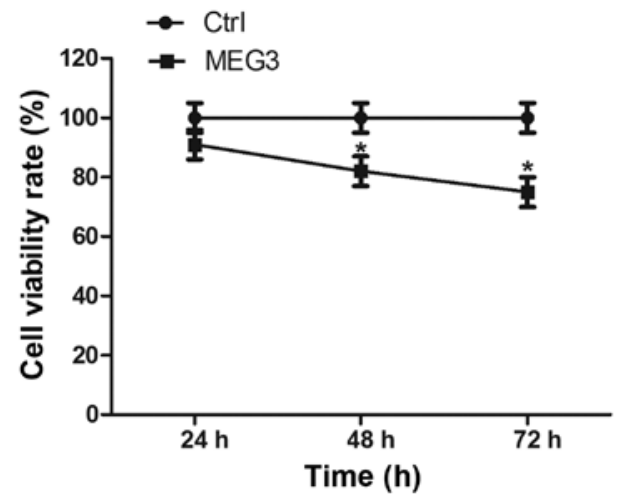

Figure 4. The effects of MEG3 on cell viability in EC109 cells. Cell viability was measured at 48 and $72 \mathrm{~h}$ after transfection by the CCK- 8 assay. The cell growth of the pcDNA3.1-MEG3 cells was decreased compared to the control. Data are presented as the means \pm SD. Independent experiments were repeated at least three times. ${ }^{*} \mathrm{P}<0.05$ vs. control.
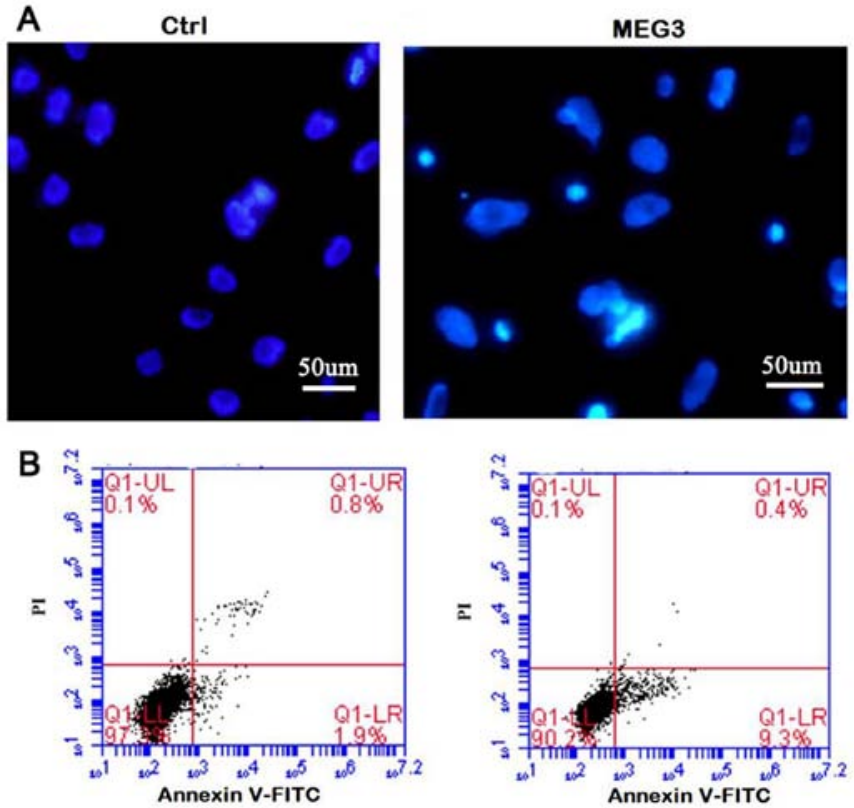

Figure 5. Effect of MEG3 on cell apoptosis. EC109 cells were transfected with pcDNA3.1-MEG3 or pcDNA3.1 and the apoptotic cells were analyzed at $48 \mathrm{~h}$ after transfection. (A) The morphology of apoptotic nuclei was observed by DAPI staining and fluorescence microscopy. (B) Apoptosis was detected by flow cytometry based on Annexin V staining (x-axis) and propidium iodide (y-axis) $(\mathrm{n}=3)$.

examine the expression of UPR and apoptosis-associated proteins CHOP, caspase-9 and cleaved caspase-3. Ectopic expression of MEG3 significantly increased the expression of molecular chaperone GRP78 and three transmembrane proteins of UPR (IRE1, PERK and ATF6), as well as CHOP, caspase-9 and cleaved caspase-3 (Fig. 6), showing that two key apoptotic pathways of ER stress (CHOP and caspase-9 pathway) were activated.

\section{Discussion}

lncRNAs were once considered to be nucleic acids without any functions and regarded as transcriptional noise. But recent
A
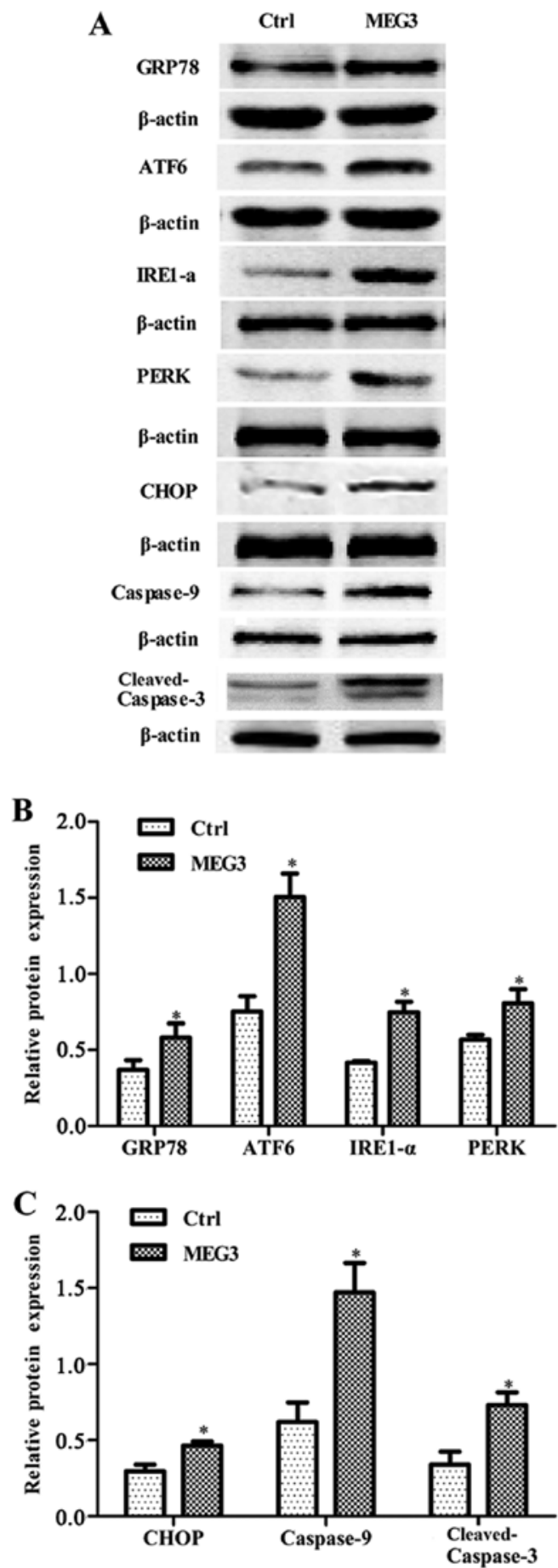

Figure 6. Effects of ectopic MEG3 on expression of ER stress-related proteins (GRP78, ATF6, IRE1- $\alpha$, PERK, CHOP, caspase-9 and cleaved-caspase-3). (A) Representative images of western blot analysis. The total cellular proteins derived from pcDNA3.1-MEG3-transfected or pcDNA3.1-transfected EC109 cells were immunoblotted with a panel of antibody specific for GRP78, ATF6, IRE1- $\alpha$, PERK, CHOP, caspase-9, cleaved caspase-3, CHOP and $\beta$-actin. (B and C) The expression of each index was normalized to expression level of $\beta$-actin, and the relative change was expressed as a ratio or fold. Data represent means $\pm \mathrm{SD}$ of three independent experiments. ${ }^{*} \mathrm{P}<0.05$ vs. control.

studies have indicated that lncRNAs play important roles in various cellular development and human diseases $(17,18)$. To date, many lncRNAs have been identified, and their involvement in all kinds of tumors has been reported. However, the molecular basis is still not well established. Therefore, the 
relationship between proteins and specific lncRNAs is a hot topic in the field of tumor biology. Although the expression of MEG3 has been shown to be downregulated in some tumor tissues (6-9), it still needs to be further confirmed in ESCC patients. The paired normal adjacent esophageal tissues were also collected and were used as the controls. The results showed that MEG3 expression was significantly decreased compared to the levels of matching normal specimens (Fig. 1). This finding was consistent with recent studies in ESCC and breast cancer $(19,20)$. Therefore, the present study once again confirmed the abnormal expression of MEG3 in ESCC.

In order to further identify the biological function of MEG3, MEG3 gene was designed according to the GenBank and synthesized by Invitrogen Life Technologies. pcDNA3.1-MEG3 plasmid vectors were constructed. The MEG3 gene was confirmed by XhoI and BamHI restriction enzyme digestion, then inserted into the pcDNA3.1 vector and transfected into EC109 cells (Fig. 2). The results showed that the MEG3 mRNA expression level is low in EC109 cells (Fig. 3) and ectopic expression of MEG3 inhibited cell proliferation (Fig. 4). Moreover, fluorescence microscopy and Annexin V/PI staining analysis confirmed that overexpressing MEG3 induced cell apoptosis (Fig. 5), which demonstrates that MEG3 functions as a tumor suppressor in EC.

Our previous study showed that adenosine, a cytotoxic drug, can cause ER stress in EC109 cells in which GRP78 displays an anti-apoptotic effect (14). In this study, we found that ectopic expression of MEG3 also increased the molecular chaperone GRP78 expression, which was consistent with our previous results in hepatoma HepG2 cells (11), showing that MEG3-induced ER stress might not be a rare phemenon in tumor cells.

As previously described, the activation of UPR starts from dissociation of GRP78 from ATF6, IRE1 and PERK. The activation of ATF6, IRE1 and PERK can enhance the degradation of unfolded and misfolded proteins through proteasomes $(14,21)$, if the stress is temporary $(22)$. However, if the cells suffer from prolonged or severe stress, additional responses are initiated, involving IRE1/Ask1/JNK, caspase-12/caspase-9/caspase-3, ERK/ATF-4/CHOP pathways and these pathways can promote cell apoptosis $(23,24)$. All three branches of the UPR regulate the activation of CHOP (25). In the present study, the expression of the three key proteins of UPR (IRE1, ATF6 and PERK) was increased in MEG3 overexpression cells. Furthermore, the expression of CHOP, caspase- 9 and cleaved caspase-3 was also upregulated in MEG3 overexpression group (Fig. 6), showing that MEG3 activates UPR downstream pathways. Overexpressing MEG3 inhibited cell growth and induced cell apoptosis (Figs. 4 and 5). Taken together, these results demonstrate that MEG3 triggers apoptosis via the ER stress pathway in EC109 cells. In our study, caspase-9 was significantly activated and the change of mitochondrial membrane potential was observed in MEG3 overexpression group (data not shown). Since caspase-9 is also an important factor of the mitochondrial apoptotic pathway, we postulate that other mechanisms may also be involved in MEG3-induced apoptosis and it need further investigation in different esophageal cancer cell lines.
In conclusion, the present study presents that MEG3 is significantly downregulated in esophageal squamous cell carcinoma tissues and exerts tumor-suppressive functions. The mechanism of MEG3-induced apoptosis may be related to the activation of the ER stress pathway.

\section{Acknowledgements}

The present study was supported by the Guangdong Natural Science Foundation in China (no. 2014A030313470) and the Collaborative and Creative Center, Molecular Diagnosis and Personalized Medicine, Shantou University, Guangdong, China. This study was also supported by the Department of Education, Guangdong Government under the Top-tier University Development Scheme for Research and Control of Infectious Diseases.

\section{References}

1. Vihinen M: Establishment of an international database for genetic variants in esophageal cancer. Ann N Y Acad Sci 1381: 45-49, 2016.

2. Gandon A, Gronnier C, Renaud F, Borde P, Vanderbeken M, Hec F, Piessen G, Adenis A, Mirabel X and Mariette C: Esophageal adenocarcinoma: Impact of a large hiatal hernia on outcomes after surgery. Ann Surg 264: 862-870, 2016.

3. Lin CY and Xu HM: Novel perspectives of long non-coding RNAs in esophageal carcinoma. Carcinogenesis 36: 1255-1262, 2015.

4. He Y, Meng XM, Huang C, Wu BM, Zhang L, Lv XW and Li J: Long noncoding RNAs: Novel insights into hepatocelluar carcinoma. Cancer Lett 344: 20-27, 2014.

5. Miyoshi N, Wagatsuma H, Wakana S, Shiroishi T, Nomura M, Aisaka K, et al: Identification of an imprinted gene, Meg3/Gt12 and its human homologue MEG3, first mapped on mouse distal chromosome 12 and human chromosome 14q. Genes Cells 5: 211-220, 2000.

6. Su L, Han D, Wu J and Huo X: Skp2 regulates non-small cell lung cancer cell growth by Meg3 and miR-3163. Tumour Biol 37: 3925-3931, 2016 .

7. Zhu J, Liu S, Ye F, Shen Y, Tie Y, Zhu J, Wei L, Jin Y, Fu H, Wu Y, et al: Long noncoding RNA MEG3 interacts with p53 protein and regulates partial p53 target genes in hepatoma cells. PLoS One 10: e0139790, 2015.

8. Sun M, Xia R, Jin F, Xu T,Liu Z, De W and Liu X: Downregulated long noncoding RNA MEG3 is associated with poor prognosis and promotes cell proliferation in gastric cancer. Tumour Biol 35: 1065-1073, 2014.

9. Ribarska T, Goering W, Droop J, Bastian KM, Ingenwerth M and Schulz WA: Deregulation of an imprinted gene network in prostate cancer. Epigenetics 9: 704-717, 2014.

10. Hu D, Su C, Jiang M, Shen Y, Shi A, Zhao F, Chen R, Shen Z, Bao J and Tang W: Fenofibrate inhibited pancreatic cancer cells proliferation via activation of p53 mediated by upregulation of LncRNA MEG3. Biochem Biophys Res Commun 471: 290-295, 2016.

11. Chen RP, Huang ZL, Liu LX, Xiang MQ, Li GP, Feng JL, Liu B and Wu LF: Involvement of endoplasmic reticulum stress and p53 in lncRNA MEG3-induced human hepatoma HepG2 cell apoptosis. Oncol Rep 36: 1649-1657, 2016.

12. Zhou Y, Zhong Y, Wang Y, Zhang X, Batista DL, Gejman R, Ansell PJ, Zhao J, Weng C and Klibanski A: Activation of p53 by MEG3 non-coding RNA. J Biol Chem 282: 24731-24742, 2007.

13. Piperi C, Adamopoulos C, Dalagiorgou G, DiamantiKandarakis E and Papavassiliou AG: Crosstalk between advanced glycation and endoplasmic reticulum stress: Emerging therapeutic targeting for metabolic diseases. J Clin Endocrinol Metab 97: 2231-2242, 2012.

14. Wu LF, Guo YT, Zhang QH, Xiang MQ, Deng W, Ye YQ, $\mathrm{Pu} \mathrm{ZJ}$, Feng JL and Huang GY: Enhanced antitumor effects of adenoviral-mediated siRNA against GRP78 gene on adenosine-induced apoptosis in human hepatoma HepG2 cells. Int J Mol Sci 15: 525-544, 2014. 
15. Zinszner H, Kuroda M, Wang X, Batchvarova N, Lightfoot RT, Remotti H, Stevens JL and Ron D: CHOP is implicated in programmed cell death in response to impaired function of the endoplasmic reticulum. Genes Dev 12: 982-995, 1998.

16. Liu LX, Deng W, Zhou XT, Chen RP, Xiang MQ, Guo YT, $\mathrm{Pu}$ ZJ, Li R, Wang GF and Wu LF: The mechanism of adenosinemediated activation of 1ncRNA MEG3 and its antitumor effects in human hepatoma cells. Int J Oncol 48: 421-429, 2016.

17. Li C, Liang G, Yao W, Sui J, Shen X, Zhang Y, Ma S, Ye Y, Zhang Z, Zhang W, et al: Differential expression profiles of long non-coding RNAs reveal potential biomarkers for identification of human gastric cancer. Oncol Rep 35: 1529-1540, 2016.

18. Ernst $\mathrm{C}$ and Morton CC: Identification and function of long non-coding RNA. Front Cell Neurosci 7: 168, 2013.

19. Lv D, Sun R, Yu Q and Zhang X: The long non-coding RNA maternally expressed gene 3 activates p53 and is downregulated in esophageal squamous cell cancer. Tumour Biol 37: 16259-16267, 2016.

20. Sun L, Li Y and Yang B: Downregulated long non-coding RNA MEG3 in breast cancer regulates proliferation, migration and invasion by depending on p53's transcriptional activity. Biochem Biophys Res Commun 478: 323-329, 2016.
21. Travers KJ, Patil CK, Wodicka L, Lockhart DJ, Weissman JS and Walter P: Functional and genomic analyses reveal an essential coordination between the unfolded protein response and ER-associated degradation. Cell 101: 249-258, 2000.

22. Lee AS: The ER chaperone and signaling regulator GRP78/BiP as a monitor of endoplasmic reticulum stress. Methods 35: 373-381, 2005.

23. Liu G, Sun Y, Li Z, Song T, Wang H, Zhang Y and Ge Z: Apoptosis induced by endoplasmic reticulum stress involved in diabetic kidney disease. Biochem Biophys Res Commun 370: 651-656, 2008.

24. Hitomi J, Katayama T, Taniguchi M, Honda A, Imaizumi K and Tohyama M: Apoptosis induced by endoplasmic reticulum stress depends on activation of caspase-3 via caspase-12. Neurosci Lett 357: 127-130, 2004.

25. Bromati CR, Lellis-Santos C, Yamanaka TS, Nogueira TC, Leonelli M, Caperuto LC, Gorjão R, Leite AR, Anhê GF and Bordin S: UPR induces transient burst of apoptosis in islets of early lactating rats through reduced AKT phosphorylation via ATF4/CHOP stimulation of TRB3 expression. Am J Physiol Regul Integr Comp Physiol 300: R92-R100, 2011. 